Article

\title{
Charging Neutral Cues with Aggressive Meaning through Violent Video Game Play
}

\section{Robert Busching * and Barbara Krahé}

Department of Psychology, University of Potsdam, Karl-Liebknecht-Straße 24/25, D-14476 Potsdam, Germany; E-Mail: krahe@uni-potsdam.de

* Author to whom correspondence should be addressed; E-Mail: busching@uni-potsdam.de; Tel.: +49-331-977-2845; Fax: +49-331-977-2795.

Received: 13 September 2013; in revised form: 30 October 2013 / Accepted: 4 November 2013 / Published: 12 November 2013

\begin{abstract}
When playing violent video games, aggressive actions are performed against the background of an originally neutral environment, and associations are formed between cues related to violence and contextual features. This experiment examined the hypothesis that neutral contextual features of a virtual environment become associated with aggressive meaning and acquire the function of primes for aggressive cognitions. Seventy-six participants were assigned to one of two violent video game conditions that varied in context (ship vs. city environment) or a control condition. Afterwards, they completed a Lexical Decision Task to measure the accessibility of aggressive cognitions in which they were primed either with ship-related or city-related words. As predicted, participants who had played the violent game in the ship environment had shorter reaction times for aggressive words following the ship primes than the city primes, whereas participants in the city condition responded faster to the aggressive words following the city primes compared to the ship primes. No parallel effect was observed for the non-aggressive targets. The findings indicate that the associations between violent and neutral cognitions learned during violent game play facilitate the accessibility of aggressive cognitions.
\end{abstract}

Keywords: media violence; aggressive cognitions; associative networks; learning

\section{Introduction}

Computer games are used to teach about a wide array of different topics, such as mathematical skills, medical procedures, or behavior in emergency situations (for a review [1]), and they are also 
used for the purposes of product advertising [2]. Employing computer games for these purposes is based on the assumption that the information presented to players during game play becomes relevant in the real world. Since aggressive content is an important part of many computer games [3], and aggressive content in a game is embedded in a wide range of contexts and backgrounds, the aim of this study was to investigate whether these originally neutral contexts become retrieval cues for aggressive cognitions after playing a violent game. According to the General Aggression Model [4], aggressive cognitions play a key role in paving the way for aggressive behavior. People for whom aggressive thoughts are easily accessible have a lower threshold for acting aggressively because they can more easily retrieve these kinds of behavior from memory [5].

Aggressive cognitions may be chronically salient or activated situationally, for example, through a provocation. In addition, aggressive behavior can be triggered indirectly through associations with other stimuli. Berkowitz and LePage's [6] finding that the mere presence of weapons leads to an increase in aggressive behavior in participants who were previously angered suggests that the presence of a stimulus associated with aggression, such as a weapon, increases aggressive behavior. Anderson, Benjamin, and Bartholow [7] demonstrated that the mere presence of weapons also leads to an increase in aggressive cognitions. However, the effect of associated cues on triggering aggressive cognitions and behavior critically depends on the meaning assigned to the cue. For example, hunters showed a "weapons effect" in terms of faster recognition and more aggressive behavior following the presentation of an assault gun, but not after the presentation of a hunting gun. Non-hunters showed faster recognition for aggressive words and more aggressive behavior after seeing pictures of hunting guns as opposed to assault guns [8]. These differences in associations between guns and aggressive cognitions are explained as a result of differences in learning histories.

While weapons are clearly part of an associative network of aggression-related concepts, neutral concepts with no explicit link to aggression, such as hot temperatures [9], African American ethnicity [10], or alcohol [11], can also cue the retrieval of aggression-related concepts, which may translate into aggressive behavior, as shown by Bargh, Chen, and Burrows [12]. They primed half of their participants with Caucasian ethnicity, while the other half was primed with African American ethnicity. After 130 trials of a boring and tedious task, a computer failure was simulated and the experimenter informed the participants that they would have to repeat the task. Participants who were primed with the African American ethnicity stereotype showed significantly more aggressive behavior in response to this provocation than those primed with the Caucasian ethnicity stereotype.

These studies are based on the concept of memory as a semantic network in which the constructs are represented as nodes and the relations between them are represented as associations [13,14]. Any learning experience is encoded in the associations between the nodes. For hunters, the associations between hunting guns and aggression are weaker than for non-hunters because they have learned to associate hunting guns with sports.

Aggressive actions performed during video game play have been shown to function as primes that facilitate the activation of aggressive cognitions. A meta-analysis of 53 studies found an overall effect of $r=0.16$ for an increase in aggressive cognitions after playing violent video games [15]. This research assumes that the violence encountered during game play is responsible for priming aggressive thoughts. In the present study, we propose an additional mechanism through which violent video game play may promote aggressive cognitions. We predict that originally neutral cues that are encountered 
in the context of playing a violent video game will become associated with aggressive cognitions through a learning process, thereby acquiring the function of primes for aggressive cognitions outside the game context.

Learning theories predict that concepts that are repeatedly presented together become associated with each other $[14,16]$. This means that media users should learn to associate the context in which violence is presented in the media with the concept of violence. For example, in many first-person shooters it is essential to learn that oil barrels can provide cover if they are empty and they can also be a tool for killing an opponent if they are filled with oil. This means that the neutral concept of an oil barrel is associated with aggressive meaning during game play.

If this association was limited to the situation of playing computer games, no effects on aggression outside the gaming context would be expected. However, if associations acquired during game play affect thinking and behavior outside the gaming context, another pathway is identified through which violent media use may affect aggressive behavior. If the player has learned to associate oil barrels with violence, the sight of an oil barrel in everyday life might prime aggressive cognitions, which, in turn, could lower the threshold for an aggressive response.

In the present study, we investigated whether the association of violence with neutral background cues in a video game environment affects the accessibility of aggressive cognitions outside the game context. We propose that there are two mechanisms involved in the associative learning process that leads from violent video game play to aggression. The first is that the more violence people encounter during video game play, the more easily they can activate aggressive cognitions when presented with an aggressive prime. Such a priming effect of violent video game play has been demonstrated by several studies in the literature [17-19]. The second mechanism is proposed to work through changing the underlying semantic memory networks by creating an association between violent cues in the video game and neutral cues from the game context. As a result of this process, neutral cues are imbued with aggressive meaning through the association with violent content and thereby facilitate the subsequent retrieval of aggressive cognitions in the presence of the neutral cues.

To investigate these two processes, habitual media violence exposure was used to assess the long term effect, while the short term effect of increased availability of aggressive cognitions was investigated by comparing the playing of a violent computer game with a control task. To test whether initially neutral cognitions would activate aggressive cognitions, the players of the violent game were divided into two groups who played the same game in two different settings: (1) in a ship setting or (2) in a rundown city suburb. The availability of aggressive cognitions was measured using a Lexical Decision Task which included primes that were related either to cities or to ships. The proposed associative networks formed during violent video game play in the ship and the city context are illustrated in Figure 1.

The left-hand panel shows that the violent cues and the two contexts, ship and city, form independent clusters of associations. After playing a violent video game in the context of a ship, associations are assumed to be formed between the violent constructs and the ship-related constructs, as shown in the middle panel. Similarly, for participants playing the violent game in the city context, associations between violent and the city-related constructs are expected to be formed, as shown in the right panel. 
Figure 1. Illustration of semantic memory networks. The ovals represent distinct nodes, while the connecting lines between the nodes represent the associations. The thicker the lines, the more closely the nodes are connected. Prior to the experiment, there are three different distinct groups of nodes (a), after playing a level in the ship condition, the aggressive nodes become associated with ship-related related nodes (b), in the city condition the aggressive nodes become associated with city-related nodes (c).

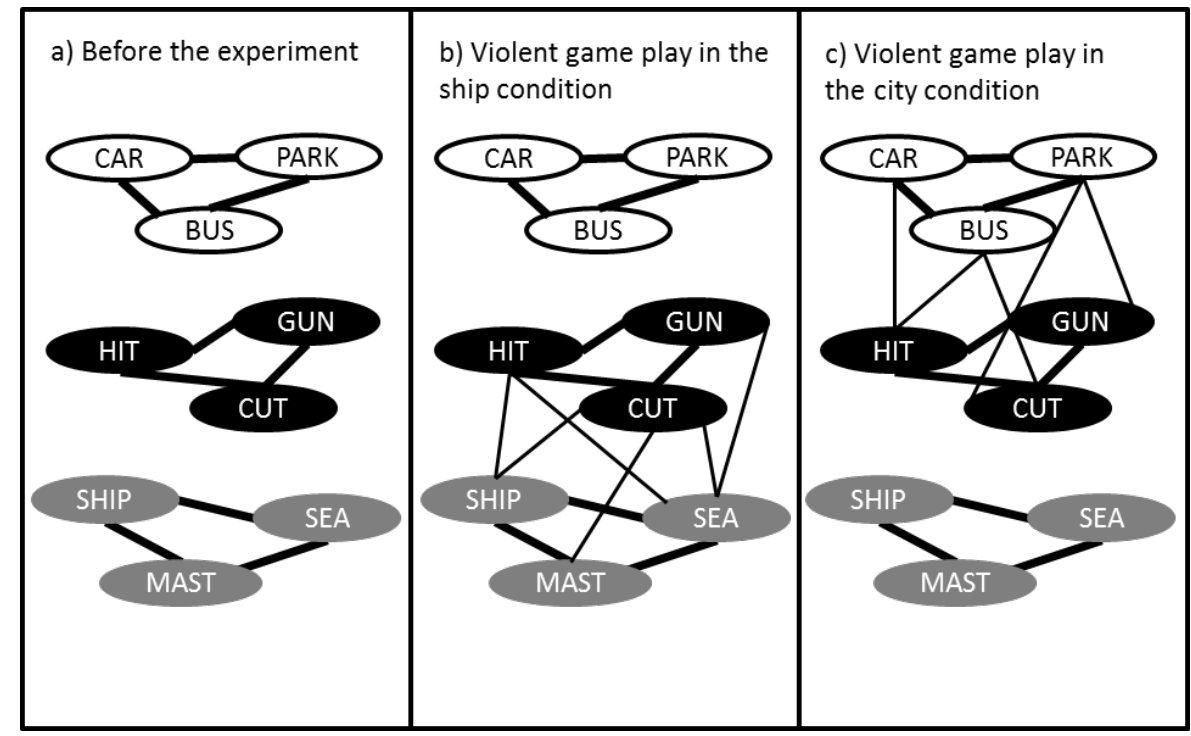

Based on this conceptualization of associative networks, three hypotheses were examined in the current study:

First, it was expected that participants with higher habitual use of violent media would respond faster to aggressive words in a Lexical Decision Task than participants with low habitual use of violent media. This hypothesis is based on the assumption that the repeated exposure to violence cues in the virtual reality of the media increases the chronic salience of aggression-related cognitions, as evidenced in shorter reaction times for recognizing words with aggressive meaning.

Second, we predicted that participants who played a violent game would respond faster to aggressive words in a subsequent Lexical Decision Task than participants in the control condition, because violent video game play is assumed to increase the situational salience of aggressive cognitions.

The third, and central, prediction referred to the proposed association of neutral cues with aggressive cognitions. It was expected that originally neutral cues in the game environment would subsequently act as primes for aggressive cognitions. Specifically, we predicted that neutral cues from the ship context, should become associated with aggressive cognitions and act as a prime for activating aggressive targets in the Lexical Decision Task for the group playing the ship level, (Figure 1b), while city-related primes should act as primes for aggressive cognitions in the city condition (Figure 1c).

\section{Method}

\subsection{Participants}

Seventy-seven male students with a mean age of $\mathrm{M}=24.74$ ( $\mathrm{SD}=4.46$ ) participated in this experiment. They were recruited via flyers distributed on campus and received either course credit or a 
10 Euro book voucher in return for participation. This study was advertised as a study investigating the relationship between media use and reaction time. Informed consent was obtained twice, once before participants registered for the online questionnaire and again before the start of the laboratory session.

\subsection{Measures}

\subsubsection{Media Violence Use}

The habitual use of media violence was assessed using the genre approach. Participants indicated on a scale from (0) never to (4) very often the frequency with which they used each of 37 genres of TV series, movies, and video games. For each genre, the frequency rating was multiplied by the violence rating assigned to the genre by independent media experts, who used a scale from (1) non-violent to (5) very violent. All genres with violence ratings of greater than $2(n=26)$ were averaged into an index of media violence use, the remaining genres with violence ratings of equal or less than $2(n=11)$ were averaged into an index of non-violent media use. This genre-based approach to measuring media violence use was found to be reliable and valid in a cross-cultural study [20].

\subsubsection{Aggressive Cognitions}

A Lexical Decision Task (LDT) was used to assess aggressive cognitions [21]. In this task, participants have to decide whether or not a given character string, the target, is a meaningful word. Before each target, a prime is presented. If the prime leads to a faster reaction time for the target than a comparison stimulus, it is assumed that the target and the prime are associated. The word and non-word targets were the same as in [22]. The words were aggressive (e.g., cannon, weapon, knives) or non-aggressive (flower, summer, meadow) in meaning, the non-words were six-letter strings (e.g., rahmin, strese, faltar). The primes were specifically chosen for this study. Since one group was assigned to play a level of a computer game set in the environment of a ship and the other group to play a level of the same game set in a city, the primes had to be related either to a ship or a city context. A pilot study was conducted to ensure that each word was only related to one of the two contexts and that none of the primes were related to aggression. ${ }^{1}$

\subsection{Procedure}

Participants first completed a short online questionnaire to assess their habitual use of violent and non-violent media, as described above. At the end of the questionnaire, they could register for the

1 The city related words were: AMPEL (traffic light), ASPHALT (asphalt), AUTO (car), BÜROS (offices), BAUSTELLE (building site), EINKAUFSZENTRUM (shopping mall), GEBÄUDE (building), GESCHÄFT (shop), HAUS (house), HOCHHAUS (skyscraper), KINO (cinema), LAUT (loud), MAUER (wall), PARKPLATZ (parking lot), S-BAHN (urban railway), SCHULE (school), SPIELPLATZ (playground), STADTPARK (city park), STRASSE (street), WERBUNG (advertisement); the ship related words were KAPITÄN (captain), FÄHRE (ferry), KAJÜTE (cabin), BORD (board), BOOT (boat), WASSER (water), SEEMANN (sailor), MEER (sea), PASSAGIER (passenger), AMPFER(steamboat), EISBERG (ice berg), MATROSE (sailor), STEUERRAD (wheel), WELLEN (waves), DECK (deck), FISCH (fish), OZEAN (ocean), SEGEL (sail), ANKER (anchor), BULLAUGE (porthole). 
second, lab-based part of the study. In the lab session, participants were randomly assigned to one of three groups. Two groups played a level of "Counter-Strike: Source”, set either on a ship or in a rundown city suburb [23]. Both conditions involved so-called fight yard levels with computer-controlled opponents, where the player had to survive as long as possible while killing as many computer-controlled opponents as possible. Each participant played a total of seven rounds, and playing sessions lasted 10 minutes on average. Participants in the control condition were asked to look at pictures of a range of different cities and indicate whether they would want to live or work in, or travel to, these cities. It was ensured that the pictures did not contain any violent action or famous landmarks. Finally, all participants completed the LDT.

\subsection{Data Preparation}

The data of one participant was excluded from the analysis due to an error rate of $50 \%$ in the LDT. The reaction time data of the remaining 76 participants were transformed using the negative inverse to ensure a normal distribution of residuals [24,25]. Since the reaction times of the non-words were not related to any psychological construct of interest in this study the reaction times of these trials were not analyzed.

\section{Results}

To test whether the habitual use of violent and non-violent media, displayed in Table 1, differed between the three experimental conditions, two ANOVAs were conducted that did not yield a significant condition effect on either violent $(\mathrm{F}(2,75)=0.89$; n.s.) or non-violent media exposure $(\mathrm{F}(2,75)=0.68$; n.s.). Additionally, it was examined whether the players' in-game behavior differed between the two game conditions. No significant difference was found in the number of opponents killed $(\mathrm{F}(1,46)=1.11$, n.s. $)$ as well as the number of times the participant got killed $(\mathrm{F}(1,46)=0.06$, n.s. $)$. Thus, randomization regarding habitual patterns of violent and non-violent media use was successful, and players encountered similar levels of violence in the two game conditions.

Table 1. Means and standard deviations (SD) for media exposure and in-game behavior.

\begin{tabular}{cccc}
\hline Variable & City Condition & Ship Condition & Control Condition \\
\hline & Mean (SD) & Mean (SD) & Mean (SD) \\
Media violence use & $7.6(1.7)$ & $7.6(1.81)$ & $8.2(2.16)$ \\
Non-violent media use & $3.6(0.8)$ & $3.7(0.68)$ & $3.8(0.95)$ \\
Number of times player was killed & $1.7(4.7)$ & $1.8(2.7)$ & \\
Number of opponents killed & $7.7(5.5)$ & $6.2(4.9)$ & \\
\hline
\end{tabular}

Because media violence exposure was a continuous predictor, a mixed effects model rather than an ANOVA with median split on the media violence use measure was employed for hypothesis testing [26]. This type of model allows us to test for interactions between continuous and discrete predictors. Discrete variables are tested by contrasts. The transformed reaction times in the LDT were the dependent variable. The target category (aggressive or non-aggressive words), the prime category (ship- or city-related), and the game context (ship, city, control) were the independent variables. All interaction terms were included. Measures of violent and non-violent media use were included as main effects and 
in interaction with the experimental factors. A Helmert contrast was used to compare the three experimental conditions. The first contrast compared the two computer game conditions with the control condition to test whether the violent content of the computer game influenced reaction times. The second contrast compared the two game conditions to establish whether the context on its own, irrespective of violence, had an effect. The coefficients from this analysis are presented in Table 2.

Table 2. Predictors of transformed reaction times.

\begin{tabular}{cc}
\hline Predictor & $\boldsymbol{B}[\mathbf{C I}]$ \\
\hline (Intercept) & $-1.38[-1.43--1.32] * * *$ \\
Target category 1 & $0.02[-0.03-0.07]$ \\
Prime category ${ }^{*}$ & $0[-0.01-0.01]$ \\
Target category $\times$ prime category & $0[-0.02-0.01]$ \\
Media violence use & $0.02[-0.02-0.06]$ \\
Non-violent media use & $-0.03[-0.07-0.02]$ \\
Target category $\times$ media violence use & $-0.02[-0.04-0.01] *$ \\
Target category $\times$ non-violent media use & $0.01[0.01-0.03]$ \\
Condition contrast $1^{3}$ & $0.02[-0.01-0.04]$ \\
Condition contrast $2^{4}$ & $0.01[-0.04-0.06]$ \\
Target category $\times$ condition contrast 1 & $0.01[0.00-0.02]$ \\
Target category $\times$ condition contrast 2 & $0.00[-0.02-0.01]$ \\
Prime category $\times$ condition contrast 1 & $0.00[-0.01-0.01]$ \\
Prime category $\times$ condition contrast 2 & $-0.01[-0.02-0.01]$ \\
Target category $\times$ prime category $\times$ condition contrast 1 & $0.00[-0.01-0.01]$ \\
Target category $\times$ prime category $\times$ condition contrast 2 & $0.02[0.01-0.04] *$ \\
\hline
\end{tabular}

Note: ${ }^{1}$ Target category dummy coded $0=$ non-aggressive target, $1=$ aggressive target; ${ }^{2}$ prime category effect coded $-1=$ ship, $1=$ city; ${ }^{3}$ Condition Contrast $1=$ comparing in the mean of the two violent conditions with the mean of the control condition; ${ }^{4}$ Condition Contrast $2=$ comparing the two different game conditions, square brackets are $95 \%$ confidence intervals; * $p<0.05 ; * * * p<0.001$.

The first hypothesis predicted an increased availability of aggressive cognitions among participants scoring high on habitual use of violent media. The interaction between media violence use and target category indicated significantly shorter reaction times to aggressive target words the more participants habitually used violent media ( $\mathrm{b}=-0.02$; $\mathrm{CI}=[-0.02--0.01] ; p<0.05)$, whereas no effect was found on the non-aggressive target words. This effect, confirming Hypothesis 1, is displayed in Figure 2. The situational priming effect of playing a violent video game predicted in Hypothesis 2 was not found. The first condition contrast, which compared the average of the two violent game conditions with the control condition, did not show significantly shorter reaction times after violent game play compared to the control condition ( $\mathrm{b}=0.00$; $\mathrm{CI}=[-0.01-0.01]$; n.s.).

The central hypothesis of this study referred to the comparison of the two game conditions. It was expected that participants who had played the city level would subsequently respond faster to aggressive targets preceded by city primes rather than by ship primes, whereas participants in the ship condition would respond faster to aggressive targets preceded by ship primes rather than by city primes. No parallel effects should occur for the non-aggressive words. The three-way interaction of target category, prime category, and ship versus city condition predicted in this hypothesis was 
significant $(\mathrm{b}=0.02 ; \mathrm{CI}=[0.01-0.04], p<0.05)$. As displayed in Figure 3, the reaction times for aggressive target words were shorter for participants in the ship condition after a ship prime compared to a city prime. The opposite pattern was found for the city condition: Participants in the city condition had shorter reaction times after the city primes than after the ship primes. No effects of the different primes were found on the non-aggressive target words. This means that the originally neutral context cues became associated with aggressive cognitions, turning them into primes that facilitated the accessibility of aggressive target words.

Figure 2. Interaction between habitual media violence use and target category on reaction times for aggressive target words.

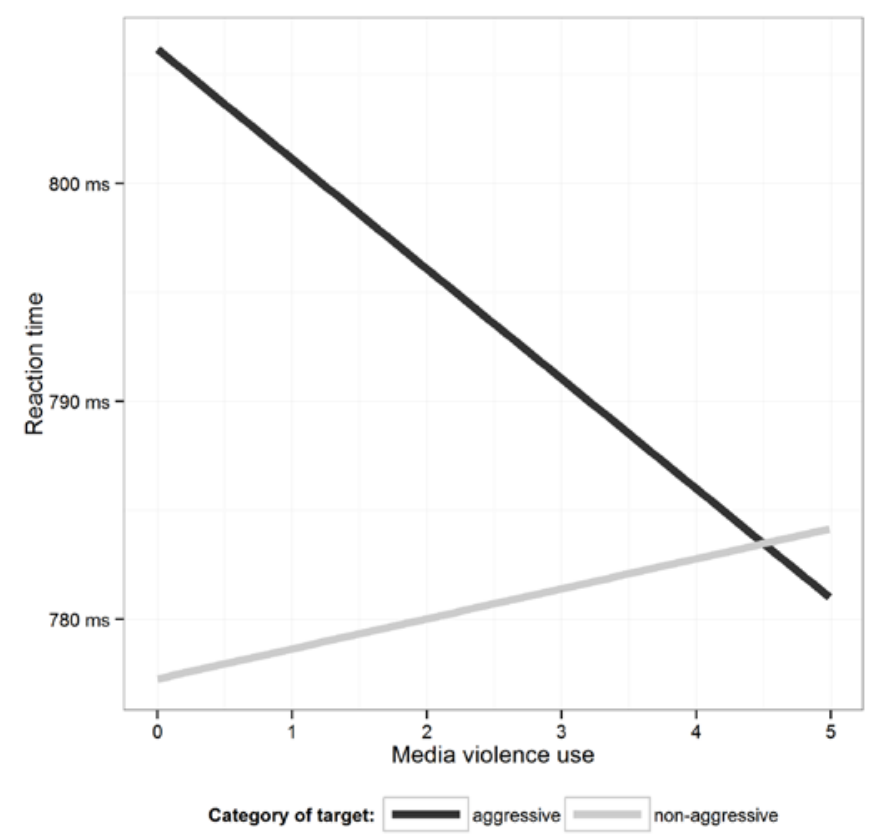

Figure 3. Reaction times in the Lexical Decision Task (LDT) depending on condition, category of prime, and category of target.

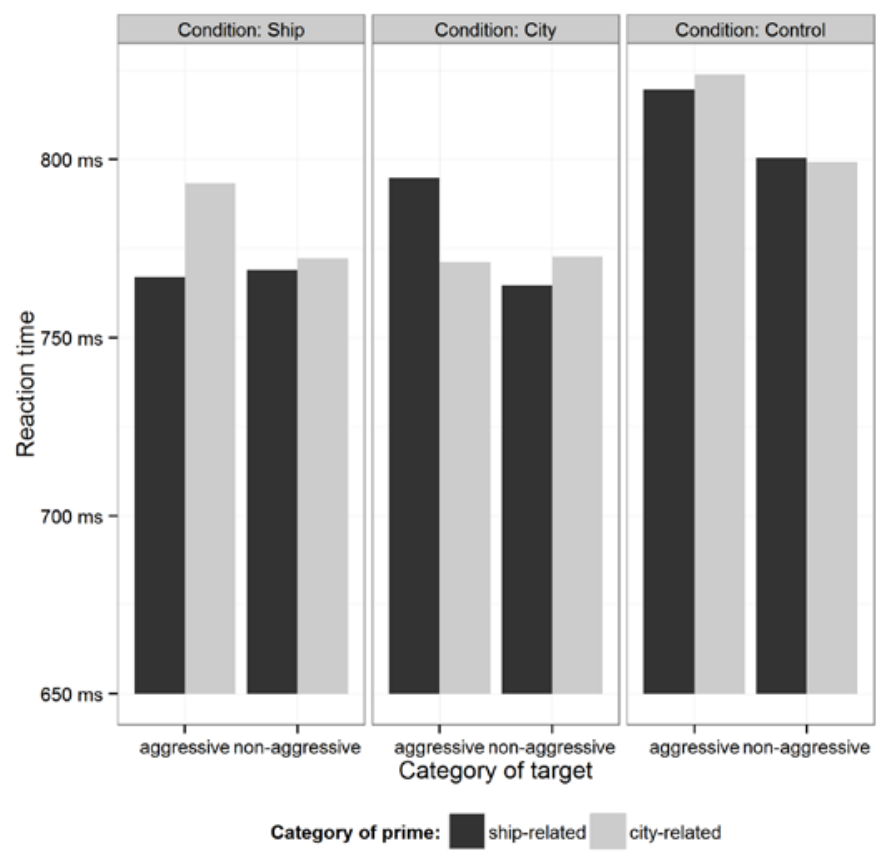




\section{Discussion}

This experiment examined the impact of violent video game play on the accessibility of aggressive cognitions as measured in a Lexical Decision Task. First, it was found that the more time participants habitually spent using violent media, the faster they were at identifying aggression-related words in a Lexical Decision Task. This finding suggests that habitual use of violent media increases the salience of aggressive thoughts, which is reflected in a higher accessibility of aggressive cognitions. The second hypothesis predicted a parallel effect for participants in the two violent game conditions, for whom the situational salience of aggressive thoughts should have been increased through playing the violent game, also leading to faster reaction times for recognizing aggressive words in the Lexical Decision Task. This effect, however, was not found in the present data. One possible explanation is that the control condition may have also activated aggressive cognitions to a certain extent. Although care was taken that the pictures did not contain any violence, participants may have thought about the potential risk of becoming a victim of crime when considering whether or not they would like to live in the different cities. To rule out this possibility, the findings need to be replicated using a different control condition with no possible association to the themes of crime and violence.

The key mechanism proposed and tested in this research referred to the association of neutral cues from the game context with violence-related constructs elicited by the actions in the game. As predicted, the neutral contexts of the ship and the city became associated with aggressive meaning, as demonstrated by the finding that ship- and city-related words served as primes for aggressive thoughts in the subsequent Lexical Decision Task.

These findings have both conceptual and methodological implications for research on aggressive cognitions in relation to media violence use. On a theoretical level, the present results show that the impact of violent media is, at least partially, governed by simple learning rules. While most theories seeking to explain the impact of violent media use on aggressive behavior $[4,18,27]$ refer to learning principles, it seems fruitful to broaden the scope beyond the direct effects of associating in-game violence with aggressive stimuli outside the game context. The indirect effects of associating violent cues with neutral cues within the game on promoting the activation of aggressive cognitions outside the game represent an additional pathway. That neutral cues may become primes of aggressive thoughts outside the game may explain why heavy users of violent media are prone to developing a hostile attributional style, interpreting ambiguous behaviors as more hostile $[28,29]$ even without the situational presence of aggressive cues. This transfer effect is likely to be stronger the more realistically the game environment is designed.

While we are not aware of any previous studies on the association of neutral stimuli with aggressive cognitions, one study has demonstrated such an association for aggressive behavior [30]. In the experiment, participants watched either a violent or a non-violent movie, both starring Kirk Douglas. Afterwards, they had the opportunity of administering electric shocks to a confederate who was either called Kirk or Bob. In the violent movie condition, the participants administered more electric shocks to the confederate called Kirk than to the confederate called Bob. No difference was found between the two names in the non-violent movie condition. This effect can be explained by the process of associative learning demonstrated in our study. The originally neutral stimulus Kirk had become associated with aggressive cognitions in the violent movie condition. 
Three limitations of the present study need to be mentioned. One is that the priming effects of originally neutral cues on aggressive cognitions were not studied in relation to a measure of aggressive behavior. As a next step, evidence is required that the formation of associations between neutral and aggressive cognitions may act as the mediating mechanism between media violence use and aggressive behavior as shown in [30]. The other limitation is that no significant effect of the violent game play conditions on reaction times for identifying the aggressive words could be found. One possible explanation is that the comparison task also activated aggressive cognitions since the control condition used images of large cities, which may have been associated with higher crime rates. Future studies should rule out this possibility by presenting images of rural areas or small towns or use neutral material without any implicit reference to the themes of crime and violence. Another explanation of this effect would be that some participants in the control condition may have been annoyed that they were not allowed to play a computer game. Although the study was not advertised as involving the playing of a computer game, it is possible that the participants in the control condition may have talked to participants in the two video game play conditions prior to participation.

The final limitation is the need for replication of the association between neutral cues and aggressive cognitions. While there is some research showing that neutral stimuli can trigger aggressive behavior [30], no other study has investigated this association. Since only one first person shooter with two different levels was used in the present study, future studies are needed that replicate the effect across other media contexts.

\section{Conclusions}

This study has shown that the habitual use of violent media predicts faster accessibility of aggressive cognitions in a laboratory task. In addition, playing a violent video game associates neutral cues with aggressive cognitions so that they acquire the status of triggers, or primes, for aggressive thoughts outside the game context. This implies that the boundary between aggressive and non-aggressive concepts becomes weaker. Playing violent video games, according to our findings, may produce associations between neutral and aggressive cognitions, imbuing the neutral cognitions with aggressive meaning.

\section{Acknowledgments}

The authors would like to thank Claudia Ahlert, Jana Haid, Marianne Hannuschke, Fabian Kirsch, and Isabell Schuster for their support in the data collection.

\section{Conflicts of Interest}

The authors declare no conflict of interest.

\section{References}

1. Connolly, T.M.; Boyle, E.A.; MacArthur, E.; Hainey, T.; Boyle, J.M. A systematic literature review of empirical evidence on computer games and serious games. Comput. Educ. 2012, 59, 661-686. 
2. Gross, M.L. Advergames and the effects of game-product congruity. Comput. Hum. Behav. 2010, 26, 1259-1265.

3. Smith, S.L.; Lachlan, K.; Tamborini, R. Popular video games: Quantifying the presentation of violence and its context. J. Broadcast. Electron. 2003, 47, 58-76.

4. Anderson, C.A.; Bushman, B.J. Human aggression. Annu. Rev. Psychol. 2002, 53, 27-51.

5. Todorov, A.; Bargh, J.A. Automatic sources of aggression. Aggress. Violent Behav. 2002, 7, 53-68.

6. Berkowitz, L.; LePage, A. Weapons as aggression-eliciting stimuli. J. Pers. Soc. Psychol. 1967, 7, 202-207.

7. Anderson, C.A.; Benjamin, A.J., Jr.; Bartholow, B.D. Does the gun pull the trigger? Automatic priming effects of weapon pictures and weapon names. Psychol. Sci. 1998, 9, 308-314.

8. Bartholow, B.D.; Anderson, C.A.; Carnagey, N.L.; Arlin, B.J.J. Interactive effects of life experience and situational cues on aggression: The weapons priming effect in hunters and nonhunters. J. Exp. Soc. Psychol. 2005, 41, 48-60.

9. De Wall, C.N.; Bushman, B.J. Hot under the collar in a lukewarm environment: Words associated with hot temperature increase aggressive thoughts and hostile perceptions. J. Exp. Soc. Psychol. 2009, 45, 1045-1047.

10. Devine, P.G. Stereotypes and prejudice: Their automatic and controlled components. J. Pers. Soc. Psychol. 1989, 56, 5-18.

11. Bartholow, B.D.; Heinz, A. Alcohol and aggression without consumption: Alcohol cues, aggressive thoughts, and hostile perception bias. Psychol. Sci. 2006, 17, 30-37.

12. Bargh, J.A.; Chen, M.; Burrows, L. Automaticity of social behavior: Direct effects of trait construct and stereotype activation on action. J. Pers. Soc. Psychol. 1996, 71, 230-244.

13. Van Overwalle, F. Introduction and overview. In Social Connectionism: A Reader and Handbook for Simulations; Van Overwalle, F., Ed.; Psychology Press: Hove, UK, New York, NY, USA, 2007.

14. Bower, G.H. Mood and memory. Am. Psychol. 1981, 36, 129-148.

15. Anderson, C.A.; Shibuya, A.; Ihori, N.; Swing, E.L.; Bushman, B.J.; Sakamoto, A.; Rothstein, H.R.; Saleem, M. Violent video game effects on aggression, empathy, and prosocial behavior in Eastern and Western countries: A meta-analytic review. Psychol. Bull. 2010, 136, 151-173.

16. Berkowitz, L. Aggression: Its Causes, Consequences, and Control; Temple University Press: Philadelphia, PA, USA, 1993.

17. Bösche, W. Violent video games prime both aggressive and positive cognitions. J Media Psychol. 2010, 22, 139-146.

18. Carnagey, N.L.; Anderson, C.A. The effects of reward and punishment in violent video games on aggressive affect, cognition, and behavior. Psychol. Sci. 2005, 16, 882-889.

19. Anderson, C.A.; Carnagey, N.L. Causal effects of violent sports video games on aggression: Is it competitiveness or violent content? J. Exp. Soc. Psychol. 2009, 45, 731-739.

20. Busching, R.; Gentile, D.; Krahé, B.; Möller, I.; Khoo, A.; Walsh, D.A.; Anderson, C.A. Testing the reliability and validity of different measures of violent video game use in the USA, Singapore, and Germany. Psych. Pop. Media Cult. 2013, doi:10.1037/ppm0000004.

21. Neely, J. Semantic Priming Effects in Visual Word Recognition: A Selective Review of Current Findings and Theories. In Basic Processes in Reading: Visual Word Recognition; Besner, D., Humphreys, G.W., Eds.; L. Erlbaum Associates: Hillsdale, NJ, USA, 1991. 
22. Krahé, B.; Möller, I.; Huesmann, L.R.; Kirwil, L.; Felber, J.; Berger, A. Desensitization to media violence: Links with habitual media violence exposure, aggressive cognitions, and aggressive behavior. J. Pers. Soc. Psychol. 2011, 100, 630-646.

23. Valve Corporation. Counter-Strike: Source [Video Game]; Electronic Arts (EA): Bellevue, WA, USA, 2011.

24. Kinoshita, S.; Mozer, M.C.; Forster, K.I. Dynamic adaptation to history of trial difficulty explains the effect of congruency proportion on masked priming. J. Exp. Psychol. Gen. 2011, 140, 622-636.

25. Kliegl, R.; Masson, M.E.J.; Richter, E.M. A linear mixed model analysis of masked repetition priming. Vis. Cogn. 2010, 18, 655-681.

26. Gelman, A.B.; Hill, J. Data Analysis Using Regression and Multilevel/Hierarchical Models, 1st ed.; Cambridge University Press: Cambridge, NY, USA, 2007.

27. Huesmann, L.R. An information processing model for the development of aggression. Aggr. Behav. 1988, 14, 13-24.

28. Krahé, B.; Möller, I. Playing violent electronic games, hostile attribution bias and aggression-related norms in German adolescents. J. Adolescence 2004, 27, 53-69.

29. Kirsh, S.J. Seeing the world through mortal kombat-colored glasses: Violent video games and the development of a short-term hostile attribution bias. Childhood 1998, 5, 177-184.

30. Berkowitz, L.; Geen, R.G. Film violence and the cue properties of available targets. J. Pers. Soc. Psychol. 1966, 3, 525-530.

(C) 2013 by the authors; licensee MDPI, Basel, Switzerland. This article is an open access article distributed under the terms and conditions of the Creative Commons Attribution license (http://creativecommons.org/licenses/by/3.0/). 